\title{
Use of microscopy techniques in failure analysis of the plastic injection molded parts to prevent the risks of serial defects in the assembly processes
}

\author{
Ion Cristian Braga ${ }^{*}$, Razvan Udroiu, and Anisor Nedelcu \\ Transilvania University of Brasov, Manufacturing Engineering Department, Mihai Viteazu No.5, \\ 500174, Brasov, Romania
}

\begin{abstract}
Nowadays, the plastic injected molded parts are components used in the automobile for both exterior and interior. A mechatronic device uses those molded parts for actuating in order to reach some effect; therefore they should be durable and aesthetically satisfying the customer requirements. The aim of this paper is to present the use of microscopy techniques in failure analysis of plastic molded parts as part of a mechatronic device in order to prevent the risks of cracking occurred in the serial assembly production. Due to the constraints, the thin walls are used in some small plastic parts and they can be broken in the next assembly steps, but the detection of the defects is not facile. At the validation of the batch, the sampling parts are tested to simulate the breakage and the tear zone is investigated using microscopy techniques. The information is used as a feedback to adjust the injection parameters, reference samples being created by experiments to support the team to reduce the time for adjusting the parameters.
\end{abstract}

\section{Introduction}

Year by year, the automotive industry is challenged to drastically reduce emissions. Speaking of Europe for instance, in regard to diesel engines, the emission control related cost increased more than three times for the EURO 6 limits compared with EURO 3 [1] as is presented in Figure 1. And the emissions are linked with the weight of the vehicle, as this is linked with the engine power. Therefore, the automakers are using more and more the alternative materials to metals in order to reduce the weight of the vehicles, so the plastic materials are one of the choices. In Europe, according to Plastics Europe, out of the demands of 47 million tons, $8.9 \%$ are used in the automotive industry [2].

\footnotetext{
* Corresponding author: jbc69mc@gmail.com
} 


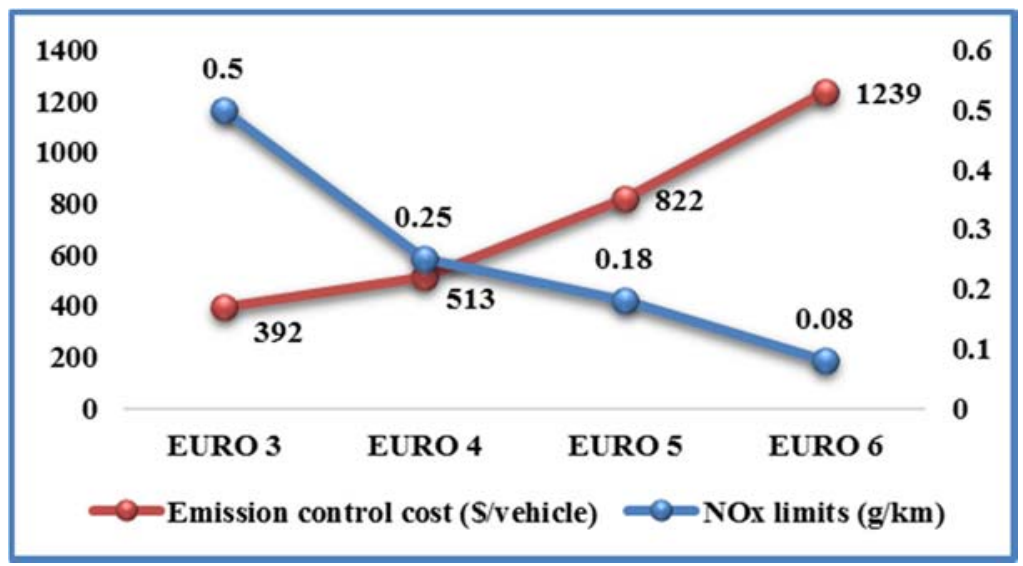

Fig. 1. $\mathrm{NO}_{\mathrm{x}}$ limits of diesel cars related emission control cost.

The plastic parts are used in different areas of the vehicles, starting with the exterior parts as bumpers, headlight housings, side mirror housings, front grille, wheel covers, panoramic roof, etc. or interior parts as door panels, center console, and instrument panel.

In regard to the technologies used to manufacture the plastic materials as automotive components could be mentioned extrusion, blow molding, injection molding, compression molding, thermoforming, vacuum forming.

The purpose of this paper is to present the failure analysis of plastic part manufactured in the plastic injection molding, the part being a part of a mechatronic device. Nowadays, the mechatronic devices used in the vehicles become smaller and the haptic feedback requested is more sensitive, therefore the paper present the risk prevention of the cracks occurrence at assembly of the plastic molded parts by using the microscopy techniques at validation of the batches and use the feedback in the plastic injection molding department to adjust properly the injection molding parameters.

\section{Plastic injection molding}

Injection molding is a process consists of heating a plastic material until become soft enough and force it into a closed mold, then by cooling down the material will be solidified and will form the specific product (Figure 2).

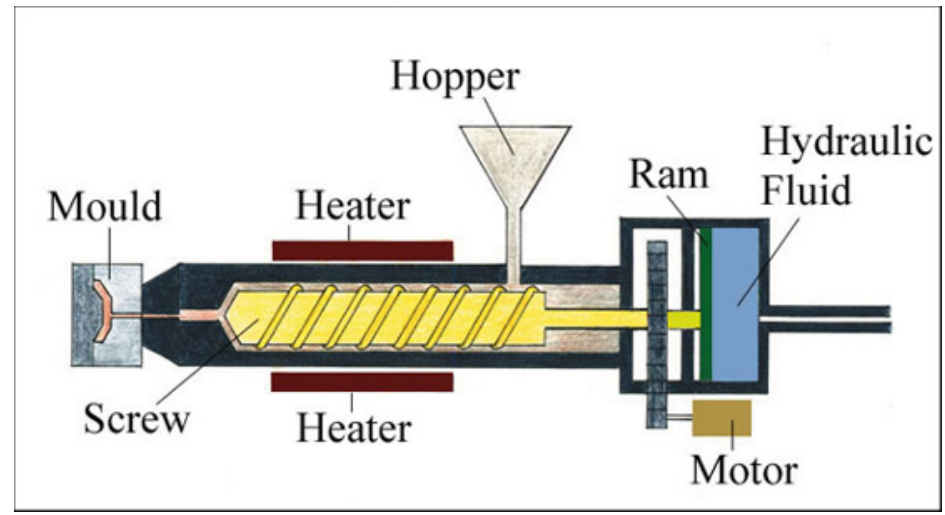

Fig. 2. Injection molding machine [3]. 
There are many parameters which must be controlled during the process in order to assure the quality level of the parts, but also the optimal cost, and these parameters could be split into four major categories: pressure, distance, time, and temperature. The best setting for these parameters depends on the material used in the molding injection and of the type of mold, as well as the status of the injection machines and, also the same importance, the environmental conditions [4].

\section{Case Study}

The study case is made on the cylindrical injection molded part which is assembled in a mechatronic device over the other cylindrical injection molded part. The assembly is made by pushing the studied part on the other part until the snap windows are secured in the three hooks positioned at 120 degrees. The thin wall is less than $1 \mathrm{MM}$, the load by snapping comes from "inside" (Figure 3).

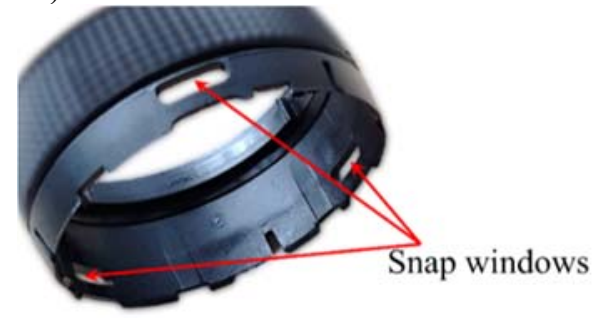

Fig. 3. Snap windows of the molded part.

The plastic material used is a composite material, an acrylonitrile-styrene-acrylate (ASA) and polyamide (PA) blend, which have a big resistance to scratches, impact resistant as well. As in the most of all plastic injection molding processes, the validation of the batches is made by measuring some dedicated dimensions established from the design phase, regular a diameter and a height, also the weight of the part. The parameters and the setting of these parameters are carefully established from the design phase and proofed by the capability of the processes and by the validation tests as well. As can be seen in figure 3, yellow marked is one of three injection points which are positioned at 120 degrees, so are equally arranged in relation to each snap window.

Currently, the start of production is made by measuring some specific dimensions plus the weight of 5 samples at the beginning of the batch and every 4 hours. The parts are taken by the quality shift responsible and measured in the laboratory; if the dimensions and the weight are according with the requirements then the process is released. But in the serial production, the variation of the parameters or the changes in the processes can lead to some risks, the small cracking of the material can occur on the thin wall of the areas near the snap windows. And this will occur at the time of the assembly when the detection of these cracks will be very low, therefore the risk to deliver these assemblies in the next operation or even to the vehicle assembly line will increase the cost of quality.

The root causes investigation is not easily to be made, so the microscopy technique was choosing to support in the analysis. In fact, the samples of the parts with small creaks of the thin wall were studied at microscope and base of the analysis of the images the brittleness of the material was detected (Figure 4). The measurements are done immediately after injection molding, the parts are kept some minutes in the laboratory conditions for the accuracy of the measurements, so there is not an influence of the moisture or temperature. 


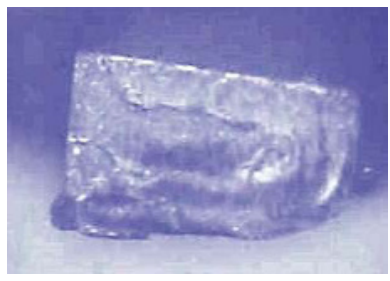

$2 \mathrm{x}$

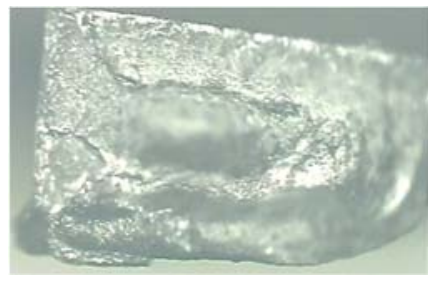

$5 x$

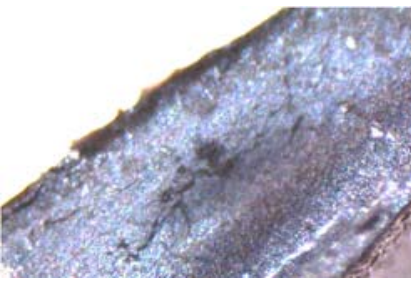

$10 \mathrm{x}$

Fig. 4. Microscopy images of the thin wall cracks with objective lens of $2 x, 5 x$ and 10x.

Based on the factors detected, as the material used was not changed, the dimensions measured and monitored with the statistical techniques shows not a significant variation, either on the studied part nor of the mating part, obviously, the combination of the parameters in injection molding are not the right one.

To implement this study in the batch validation, some experiments were developed to find the relation between the small crack occurrence and the value of the pull force in the middle of the thin wall area, as can be seen in Figure 5.

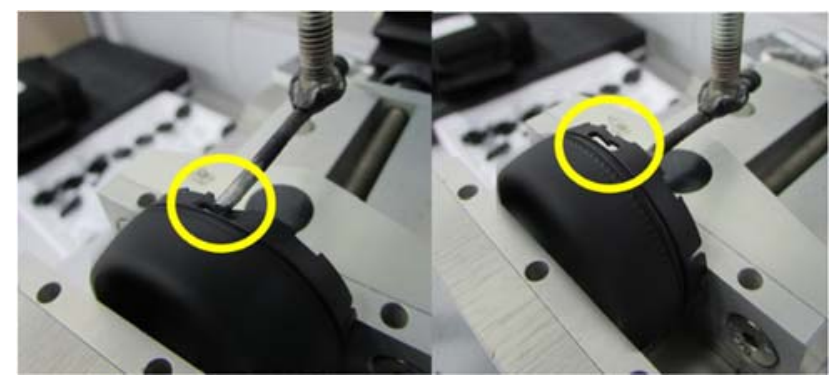

Fig. 5. Pull force measurement experiments.

The pull force measurements were conducted on each of three thin wall areas from each cavity out of the mold (four cavities for the studied part) until the thin wall was broken or until a certain maximum value of the force (for the cases when the part start to be deformed, but not broken at the maximum force). The number of the measurements was 5 shots from each cavity, and the average of the measurements can be seen in Table 1 . The minimum value measured was $8.59 \mathrm{~N}$ and the maximum was the $21.16 \mathrm{~N}$, but the mode was almost near the average of these 60 measurements. More, in the assembly processes were checked 50 pieces of sub-assemblies to detect the cracking of the thin wall due to snapping and the result was around $35 \%$ of broken parts (each sub-assembly have 3 injected parts used, so there were tested $150 \mathrm{pcs}$ ).

Table 1. Average of the pull force measurements of each cavity.

\begin{tabular}{|c|c|c|c|c|}
\cline { 2 - 5 } \multicolumn{1}{c|}{} & \multicolumn{4}{c|}{ Force (N) } \\
\hline $\begin{array}{c}\text { Thin wall } \\
\text { area }\end{array}$ & Cav. 1 & Cav. 2 & Cav. 3 & Cav. 4 \\
\hline Area 1 & 17.21 & 16.89 & 17.02 & 17.15 \\
\hline Area 2 & 16.34 & 16.32 & 16.96 & 17.54 \\
\hline Area 3 & 12.48 & 17.43 & 17.43 & 16.43 \\
\hline
\end{tabular}

Using the knowledge from these experiments, next step developed was to find the optimal combination of the parameters in injection molding to prevent this brittleness of the 
parts. At the beginning, were identified the factors with the most impact in the brittleness of the parts: the moisture of the resin was choosing as impact factor, because of the water droplets that will turn to steam when heated in the injection unit, nozzle temperature was another factor, as too hot temperature will overheat the material, then the injection pressure, as the low temperature will lead to weld areas and not good filling conditions, afterward the cycle time (short cycle will not allow the proper melting of the resin), back pressure, screw speed, and injection fill rate will lead to a thermal degradation of the plastic material through increased shear heat [5-6].

Thereby, a multi-approach team with the members from the injection molding setting, quality specialists, molding specialists, after deep analysis of the possible variations and the last changes in the processes, established six parameters and their levels to be part of the experiments. As the full factorial design of experiment was not an option, due to many factors, it means too many combinations and too much time consuming, the Taguchi method is preferred to be used for the optimization of the parameters. In the presented study, the higher value of the pull force is expected, so the signal-to-noise $(\mathrm{S} / \mathrm{N})$ ratio characteristic "the-higher-the-better" will be chosen and the orthogonal array L27 (Figure 6) [7].

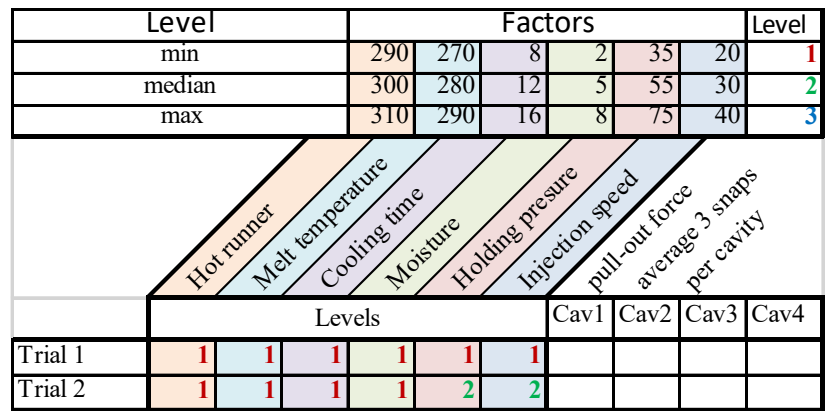

Fig. 6. Parameters and their level for Taguchi method experiments.

After each experiment, the pull force was measured on one chosen area out of three thin walls for the cavities from 1 to 4 (based on the measurements and team analysis). The average of three measurements from each cavity was transposed in the experiments table, then using a software (in this study was used Minitab software) [7-8] the optimal combination was found [9]. After the production running, the sample parts were measured out of the optimal parameter setting, and the pull force obtained, as average, was increased to $35 \mathrm{~N}$, in some cases the thin wall was deformed at $38 \mathrm{~N}$ but did not present the cracks, finally no defects were found after changing of the parameters.

The cracking area was again studied under the microscope and the fractured areas were not observed anymore (Figure 7).

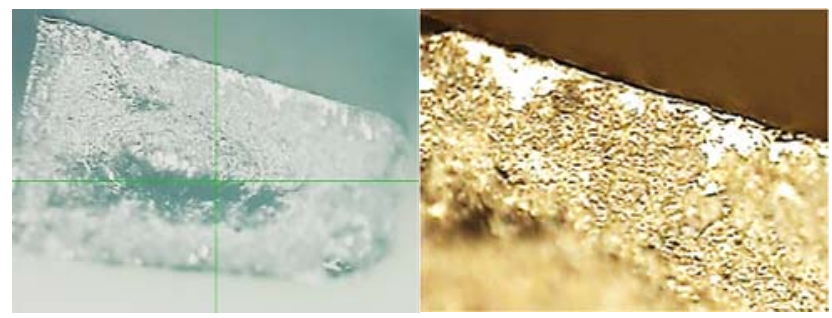

Fig. 7. Microscopy images of the thin wall cracks after optimization (with objective lens of $2 x$ and $10 \mathrm{x})$. 


\section{Conclusions}

Using of the microscopy techniques in failure analysis of the plastic injection molded products as part of the validation control of the batches in the serial production will prevent the risks of occurrence of cracks or other similar defects in further assembly processes. Based on the type of the material and the desired shape and structure of the part, a failure catalog will support the inspector to detect if there is an unexpected effect and to react accordingly.

The paper presents the solution to reduce the poor-quality cost or even the possible complaints from the customer generated by the occurrence of small cracks in plastic molding injection parts only by implementing some supplementary tests made on the already established sample for validation of the processes in injection molding. The pull force measurements and microscopy techniques will lose way less time and cost as the waste generated by the non-conform product that can pass the assembly tests. Thus, the update of the control plan with the measurements to be made, also with the value of the characteristics and method, sampling, frequency, and reaction plan it is expected.

As the plastic materials are more often used in the automotive industry as an alternative to the heavy materials, new plastic material will bring also new challenges in serial production, therefore, the Taguchi method could be used to find the optimal combination of the parameters after the new variations or changes implemented in the injection molding processes.

Last but not least, the combination of the standard validation of the start of production batches by measuring of some dimensions and the weight of material with the pull tests and/or microscopy techniques will lead to lean manufacturing processes and to a high-quality level of the products.

\section{References}

1. F. Arena, D. Spera, W.-D. Hoppe, H. Suzuki, E. Kirstetter, The future of diesel engines, http://www.adlittle.com, (2016)

2. http://www.plasticseurope.org, (2016)

3. http://www.design-technology.org/.

4. D. M. Bryce, Plastic Injection Molding...material selection and product design fundamentals, IPLAS, (2016)

5. http://plastictroubleshooter.com, (2017);

6. D. V. Rosato, D. V. Rosato, M. G. Rosato, Injection Molding Handbook, $\mathbf{3}^{\text {rd }}$ ed., (Kluwer Academic Publishers, 2000)

7. https://www.minitab.com/en-us/products/minitab/, (2016)

8. G. Taguchi, Introduction to quality engineering, Mc Graw Hill, New York (1990)

9. I.C. Braga, A. Nedelcu, Optimization of the Plastic Injection Molding Parameters by using the Taguchi Method to Prevent Jammed Components in the Assembly Process of Mechatronic Devices, CONAT 2016, 575, (2016) 